\title{
Influence of Thiol Capping on the Exciton Luminescence and Decay Kinetics of CdTe and CdSe Quantum Dots
}

\author{
Sander F. Wuister,* Celso de Mello Donegá, and Andries Meijerink \\ Condensed Matter and Interfaces, Debye Institute, Utrecht University, P.O. Box 80 000, \\ 3508 TA Utrecht, The Netherlands
}

Received: July 2, 2004; In Final Form: September 1, 2004

\begin{abstract}
Highly luminescent CdSe and CdTe quantum dots (QDs) are prepared in a hot solvent of capping molecules (TOP/TOPO/HDA for CdSe and TOP/DDA for CdTe). The influence of exchange of the capping molecules with different types of thiol molecules (amino ethanethiol, (3-mercaptopropyl)trimethoxysilane, hexanethiol, 2-propenethiol, and 4-mercaptophenol) is investigated for both CdSe and CdTe QDs. A remarkable difference is observed: capping exchange with thiol molecules results in an increased luminescence efficiency for CdTe QDs but induces quenching of the excitonic emission of CdSe QDs. The striking difference between the two types of II-VI QDs is explained by the difference in the energy of the valence band top. The lower energetic position of the valence band for CdSe results in hole trapping of the photogenerated hole on the thiol molecule, thus quenching the luminescence. For CdTe the valence band is situated at higher energies with respect to the redox level of most thiols, thus inhibiting hole trapping and maintaining a high luminescence efficiency.
\end{abstract}

\section{Introduction}

Nanocrystalline semiconductors have attracted great interest over the past decades because their optical and electrical properties are remarkably different from those of bulk semiconductors. ${ }^{1,2}$ Good control over the particle size, together with a high quantum efficiency $(\mathrm{QE})$, can be obtained by several high-temperature syntheses in coordinating solvents. ${ }^{3-5}$ A high QE, especially in the visible, is crucial for the application of quantum dots (QDs) in biological labels, quantum dot lasers, and LEDs. ${ }^{6-9}$

Thiols form an important class of capping molecules, and thiols with a polar headgroup are used to synthesize watersoluble QDs that can serve as biological labels. ${ }^{10,11}$ In the case of CdSe QDs, however, the QE strongly decreases after capping exchange with thiols such as (3-mercaptopropyl)trimethoxysilane (MPS) ${ }^{6}$ and $N, N$-dimethyl-2-mercaptoethylammonium chloride. ${ }^{12}$ In contrast we have recently prepared water-soluble, thiolcapped CdTe QDs with a high efficiency (up to 60\%) and a monoexponential decay, ${ }^{13}$ demonstrating that the capping exchange with thiols has a beneficial effect on the quantum efficiency (QE) for CdTe QDs. Highly efficient thiol-capped CdTe QDs have also been reported by Weller and coworkers, ${ }^{14,15}$ in striking contrast with the weakly luminescent thiol-capped CdSe QDs reported by the same group. ${ }^{16}$ It is the purpose of this paper to understand the remarkable difference between the luminescence behavior of these two related semiconductors upon binding to thiols. Both types of QDs were prepared via well-known high-temperature synthesis methods. The capping molecules of trioctylphosphine (TOP) and dodecylamine (DDA)-capped CdTe and TOP/trioctylphosphineoxide (TOPO)/hexadecylamine (HDA)-capped CdSe QDs were exchanged with thiols containing different functional groups (both polar and apolar). After capping exchange the CdTe QDs show a strong excitonic emission with a monoexponential decay and

* To whom correspondence should be addressed. Phone: +31-302532207. Fax: +31-30-2532403. Email: s.f.wuister@phys.uu.nl. a higher QE. However, the same thiols that resulted in an increase of the luminescence lifetime and QE for CdTe QDs induce quenching of the luminescence of CdSe QDs. This remarkable difference between $\mathrm{CdSe}$ and $\mathrm{CdTe}$ is explained by the difference in the position of the valence band relative to the redox level of the thiol capping molecule.

\section{Experimental Section}

The syntheses of CdTe and CdSe QDs are performed under a dry argon atmosphere. Capping exchange is performed in a glovebox under nitrogen. All samples are stored in vials under inert atmosphere for luminescence and luminescence lifetime measurements.

Synthesis of CdTe Quantum Dots. Highly luminescent CdTe QDs were synthesized in a mixture of TOP/DDA (trioctylphosphine/dodecylamine) following the method reported by Wuister et al. ${ }^{17,18}$ DDA was purified and degassed by heating at $100{ }^{\circ} \mathrm{C}$ under vacuum for several hours. In a three-necked flask $10 \mathrm{~g}$ of dry DDA and $7 \mathrm{~mL}$ of TOP were heated to $50{ }^{\circ} \mathrm{C}$. To this solution were added $0.22 \mathrm{~g}(1.54 \mathrm{mmol})$ of $\mathrm{Cd}(\mathrm{Me})_{2}$ in $7 \mathrm{~mL}$ of TOP and $0.16 \mathrm{~g}(1.25 \mathrm{mmol})$ of Te powder (grain size $<250 \mu \mathrm{m}$ ). The reaction mixture was heated to $185^{\circ} \mathrm{C}$ for $2 \mathrm{~h}$, yielding orange-emitting QDs with a QE of $15 \%$ at room temperature. The final product (CdTe QDs in TOP/DDA) is a liquid at room temperature.

Synthesis of CdSe Quantum Dots. Highly luminescent CdSe QDs were prepared by the method reported by de Mello Donegá et al. ${ }^{5}$ A total of $20 \mathrm{~g}$ of TOPO, $10 \mathrm{~g}$ of HDA, and $2 \mathrm{~mL}$ of anhydrous triethylorthoformate are transferred into a $250-\mathrm{mL}$ three-necked, round-bottom flask and heated slowly to $330{ }^{\circ} \mathrm{C}$. At this point, the heating mantle was removed, and the temperature was allowed to fall. A solution of $10 \mathrm{~mL}$ of TOP containing $0.79 \mathrm{~g}$ of $\mathrm{Se}$ (as TOP-Se complex) and $0.28 \mathrm{~g}$ $\mathrm{Cd}(\mathrm{Me})_{2}$ was quickly injected into the mixture when the temperature reached $300{ }^{\circ} \mathrm{C}$. After the injection, the temperature was allowed to fall further to $170{ }^{\circ} \mathrm{C}$. The reaction mixture was 
then stabilized at the desired growth temperature $\left(185^{\circ} \mathrm{C}\right)$ for $2 \mathrm{~h}$, yielding orange-emitting QDs with a QE of 55\% at room temperature.

Capping exchange with thiol molecules can be used to make QDs with surface functionalities that make them soluble in a wide variety of solvents (polar and apolar) and can give the QDs a positive or negative surface charge. The high affinity of the thiol group for the $\mathrm{Cd}$ surface atoms with respect to the amines drives the exchange of the amine (HDA or DDA) by the thiol. In the present experiments five different thiols are used with different functional groups: amino ethanethiol, (3mercaptopropyl)trimethoxysilane, hexanethiol, 2-propenethiol, and 4-mercaptophenol.

Capping Exchange of CdTe and CdSe QDs with Aminoethanethiol $\cdot \mathbf{H C l}$. Either $100 \mu \mathrm{L}$ of the final product of $\mathrm{CdTe}$ QDs in the TOP/DDA coordinating mixture or $0.2 \mathrm{~g}$ of $\mathrm{CdSe}$ in the TOP/TOPO/HDA matrix was dispersed in $5 \mathrm{~mL}$ of chloroform. Subsequently $100 \mu \mathrm{L}$ of a $0.5 \mathrm{M}$ methanolic solution of amino-ethanethiol $\cdot \mathrm{HCl}$ (AET) was added under nitrogen atmosphere until the particles flocculate. After the flocculation, ultrapure water $(16 \mathrm{M} \Omega . \mathrm{cm})$ was added to the suspension, resulting in a two-phase system. Upon shaking the QDs were transferred into the water phase, as positively charged particles. ${ }^{13}$

Capping Exchange of CdTe QDs with (3-Mercaptopropyl)trimethoxysilane. For the preparation of CdTe QDs that are soluble in ethanol, $20 \mu \mathrm{L}$ of (3-mercaptopropyl)trimethoxysilane (MPS) was added to $100 \mu \mathrm{L}$ of the final product of CdTe QDs. After $1 \mathrm{~h}$ the particles were diluted with $10 \mathrm{~mL}$ of absolute ethanol, and $100 \mu \mathrm{L}$ of $\mathrm{NH}_{3}$ (aqueous solution, 29\%) was added to hydrolyze the methoxy groups of the MPS. The optically clear solution was left overnight under stirring to complete the hydrolyzation of the MPS.

Capping Exchange of CdTe QDs with Hexanethiol. Hydrophobic thiol-capped CdTe QDs were prepared by addition of $50 \mu \mathrm{L}$ of hexanethiol to $100 \mu \mathrm{L}$ of the crude solution of CdTe QDs in the TOP/DDA coordinating mixture. After 1 day the particles were soluble in various organic solvents. For the studies reported here, chloroform $(5 \mathrm{~mL})$ was used as solvent.

Capping Exchange of CdTe and CdSe QDs with Allylmercaptan. QDs capped with an alkenylthiol were prepared by addition of $50 \mu \mathrm{L}$ of allylmercaptan (AM, 2-propenethiol) to either $100 \mu \mathrm{L}$ of the final product of CdTe QDs in the TOP/ DDA coordinating mixture or $0.2 \mathrm{~g}$ of CdSe in the TOP/TOPO/ HDA matrix. After 1 day, $5 \mathrm{~mL}$ of chloroform was added.

Capping Exchange of CdTe and CdSe QDs with 4-Mercaptophenol. QDs capped with 4-mercaptophenol were prepared by addition of $20 \mu \mathrm{g}$ of 4-mercaptophenol to either $100 \mu \mathrm{L}$ of the final product of CdTe QDs in the TOP/DDA coordinating mixture in chloroform or $0.2 \mathrm{~g}$ of $\mathrm{CdSe}$ in the TOP/TOPO/ HDA matrix in chloroform.

Apparatus. Emission spectra, recorded with a $0.25 \mathrm{~m}$ Acton Research monochromator fitted with a Princeton Instruments liquid nitrogen cooled CCD camera, and luminescence decay curves were measured simultaneously using a Pico Quant laser ( $\lambda_{\text {ex }}=406 \mathrm{~nm}, 2.5 \mathrm{MHz}$ repetition rate, $55 \mathrm{ps}$ pulse width) as excitation source. For luminescence lifetime measurements a monochromator (1350 lines $\mathrm{mm}^{-1}$ grating, blazed at $500 \mathrm{~nm}$ ) in combination with a fast Hamamatsu photomultiplier tube (H5738P-01) was used for light detection. The luminescence decay curves were obtained by time correlated single photon counting (TCSPC) via time-to-amplitude conversion (TAC) with a Time Harp 100 computer card. The ratio of stop to start pulses was kept low (below 0.05) to ensure good statistics. All luminescence decay curves were measured at the maximum of

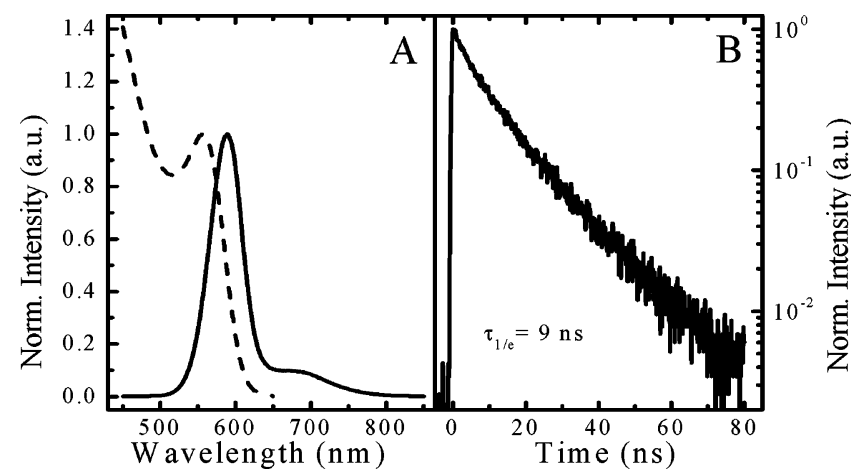

Figure 1. (A) Normalized absorption and emission spectra $\left(\lambda_{\mathrm{ex}}=\right.$ $400 \mathrm{~nm}$ ) and (B) luminescence decay curves of TOP/DDA-capped CdTe QDs.

the emission peak. The QEs of the samples were determined using a SPEX Fluorolog spectrofluorometer, equipped with two monochromators (double-grating, $0.22 \mathrm{~m}$, SPEX 1680, model F2002) and a 450-W xenon lamp as the excitation source with commercial laser dyes as standards (Rhodamine B and Coumarine $153, \mathrm{QE}=90 \%$ ), as described in detail elsewhere. ${ }^{5}$

\section{Results and Discussion}

3.1. Capping Exchange with Thiols of TOP/DDA-Capped CdTe Quantum Dots. CdTe QDs can be grown in a mixture of TOP and DDA, where TOP coordinates to Te and DDA to $\mathrm{Cd}$. Via this method CdTe QDs can be prepared at high temperatures (typically $150-200{ }^{\circ} \mathrm{C}$ ), yielding QDs with high crystallinity. The size of the particles is determined by the reaction temperature and time. Quantum efficiencies up to $65 \%$ have been obtained. ${ }^{17}$ Figure $1 \mathrm{~A}$ shows the absorption and emission spectra of CdTe QDs prepared in a mixture of TOP and DDA at a growth temperature of $185^{\circ} \mathrm{C}$. From the position of the first maximum in the absorption spectrum $(555 \mathrm{~nm})$ the particle size is estimated to be $3 \mathrm{~nm}$. The emission spectrum shows two bands. The intense band around $590 \mathrm{~nm}$ is ascribed to the exciton emission of CdTe QDs. The width of the exciton peak is $50 \mathrm{~nm}\left(1450 \mathrm{~cm}^{-1}\right)$, corresponding to a polydispersity of about $15 \%$. The broad band around $675 \mathrm{~nm}$ is ascribed to a defect-related emission. ${ }^{18}$ The decay curve of the exciton emission is depicted in Figure 1B. The decay curve is not single exponential. The faster initial decay reflects the emission from QDs with a higher nonradiative decay rate. A distribution in decay times is expected as a result of a variation in the nonradiative decay rates for different QDs due to a variation in type and number of the quenching centers. This results in a spread of luminescence lifetimes for different quantum dots. The $\tau_{1 / \mathrm{e}}$ value (the time at which the intensity has decreased to 1/e of the initial value) is an estimate for the average decay time. For TOP/DDA capped CdTe QDs (with a QE of 15\%) the decay time is determined to be about $9 \mathrm{~ns}$, which is comparable to the decay times reported in ref 15 for CdTe QDs. The features of the TOP/DDA-capped CdTe particles as described here (both excitonic and defect-related emission and a nonexponential exciton luminescence decay) are characteristic for the TOP/DDA-capped CdTe QDs that were used for the surface modification with thiols as will be discussed below.

CdTe QDs grow slowly as a result of the slow dissolution of $\mathrm{Te}$ (as TOP-Te) in the TOP/DDA mixture, thus yielding CdTe with a very high crystallinity. ${ }^{17}$ This is one of the factors contributing to the high QE. DDA capping molecules (bound to the $\mathrm{Cd}$ sites of the CdTe QD) are not very strongly bound 

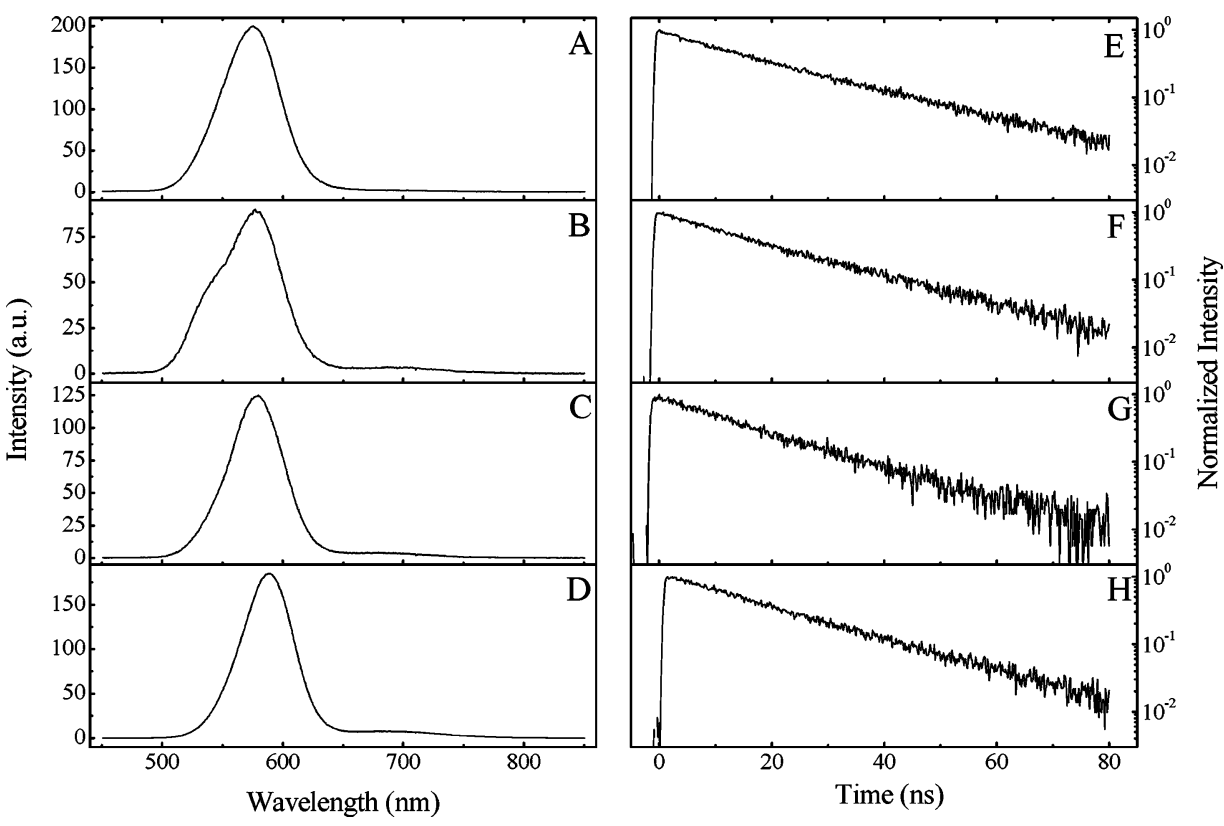

Figure 2. Emission spectra $\left(\lambda_{\mathrm{ex}}=400 \mathrm{~nm}\right)$ and normalized luminescence decay curves $\left(\lambda_{\mathrm{ex}}=406 \mathrm{~nm}\right)$ of the excitonic emission for (A) AET (in water), (B) MPS (in ethanol), (C) AM (in chloroform), and (D) HT (in chloroform) capped CdTe QDs.

capping molecules compared to, for instance, thiols. ${ }^{13}$ Therefore exchange of the DDA surface molecules for thiols takes place rapidly. This is reflected in the fast precipitation of the QDs after AET addition and the immediate increase in the luminescence intensity and the luminescence decay time upon hexanethiol addition (see below). This makes CdTe QDs, prepared in TOP/DDA, well-suited for surface molecule exchange experiments. Four different types of thiols have been used for capping exchange to make the CdTe QDs water-soluble (aminoethanethiol $\cdot \mathrm{HCl}, \mathrm{AET})$, soluble in ethanol ((3-mercaptopropyl)trimethoxysilane, MPS), and soluble in organic media by capping with saturated (hexanethiol, HT) and unsaturated (2propenethiol or allylmercaptan, AM) alkanethiols.

Figure 2 (left side) shows the emission spectra of CdTe QDs capped with AET (A), MPS (B), AM (C), and HT (D). In all four spectra an intense emission band around $590 \mathrm{~nm}$ is observed that is ascribed to the exciton emission. The exciton peak of the MPS-capped CdTe QDs has a shoulder at higher energy. The origin of this shoulder is not clear. In some of the spectra (B-D) a defect-related emission (around $680 \mathrm{~nm}$ ) is still observed, although the intensity has decreased in comparison to the intensity prior to capping exchange with thiols. This defect-related emission originates from surface defects since recapping with AET removes this type of emission almost completely. ${ }^{13}$ This defect-related emission is also observed in efficient samples since the QDs are not all chemically identical.

The contribution of the defect-related emission to the luminescence signal has reduced from $15 \%$ to at most $6 \%$ (determined by integrating the peak intensities). The QE increases after capping exchange with thiols and varies between $20 \%$ and $40 \%$.

Figure 2 (right side) shows the luminescence decay curves of CdTe QDs capped with AET (E), MPS (F), AM (G), and $\mathrm{HT}(\mathrm{H})$ measured at the maximum of the exciton peak. All luminescence decay curves show a monoexponential behavior. A monoexponential decay implies that the radiative process is dominant for all luminescing QDs. The QE of the luminescing QDs must therefore be close to unity. Because the QE of the ensemble is only $20-40 \%$, about $60-80 \%$ of the dots are assumed to be "dark" or weakly luminescent. ${ }^{13,19,20}$ For AET-,
MPS-, and HT-capped CdTe QDs the decay time was determined to be $19 \mathrm{~ns}$, and for AM-capped CdTe QDs a slightly shorter decay time was observed (17 ns). The lifetime is almost independent of the solvent and the functional group of the thiol and is close to the radiative lifetime of CdTe QDs. ${ }^{21}$ Clearly, capping exchange with thiol molecules results in a reduced nonradiative decay, as is evidenced by the increase of the luminescence QE accompanied by a single-exponential decay of the emission. The higher luminescence efficiency is ascribed to a better surface passivation by the thiol capping molecules. For CdTe QDs with an optimized QE after synthesis in TOP/ DDA, capping exchange with AET has been shown to yield water-soluble QDs with a QE as high as $60 \%^{13}$ which is in agreement with the presently observed beneficial effect of capping exchange with a variety of thiol molecules.

3.2. Capping Exchange with Thiols of TOPO/HDACapped CdSe Quantum Dots. Monodisperse CdSe QDs with high QE (up to $85 \%$ ) can be prepared in a mixture of TOP/ TOPO/HDA. ${ }^{5}$ This yields QDs with an excitonic emission band that can be tuned from green to red, depending on the growth temperature and time. Here, CdSe QDs are grown at $185^{\circ} \mathrm{C}$ for $2 \mathrm{~h}$, giving orange-emitting QDs with a QE of 55\%. Figure $3 \mathrm{~A}$ shows the emission spectrum of CdSe QDs (about $3.4 \mathrm{~nm}$ in size). The intense peak around $590 \mathrm{~nm}$ is assigned to the exciton emission. As a result of effective capping of the surface by TOP, TOPO, and HDA, no defect-related emission is observed. The luminescence decay curve is depicted in Figure $3 \mathrm{D}$. A monoexponential decay curve with a $\tau_{1 / \mathrm{e}}$ value of $28 \mathrm{~ns}$ is observed. The value of $28 \mathrm{~ns}$ for the luminescence decay time is consistent with the findings of de Mello Donegá et al. ${ }^{5}$ for a sample with an ensemble QE of $85 \%$, indicating that the emission of the CdSe QDs is dominated by radiative recombination.

For capping exchange two different thiols were used: AET and AM. Thiols are capable of partially replacing TOPO at the QD surface, ${ }^{22}$ while the amine (HDA) can be replaced efficiently by the thiol. AET-capped particles were suspended in water, and AM-capped particles in chloroform. In Figure 3 the emission spectra $(\mathrm{B}, \mathrm{C})$ and luminescence decay curves $(\mathrm{E}, \mathrm{F})$ are depicted for the CdSe QDs after capping exchange with AET and AM. 

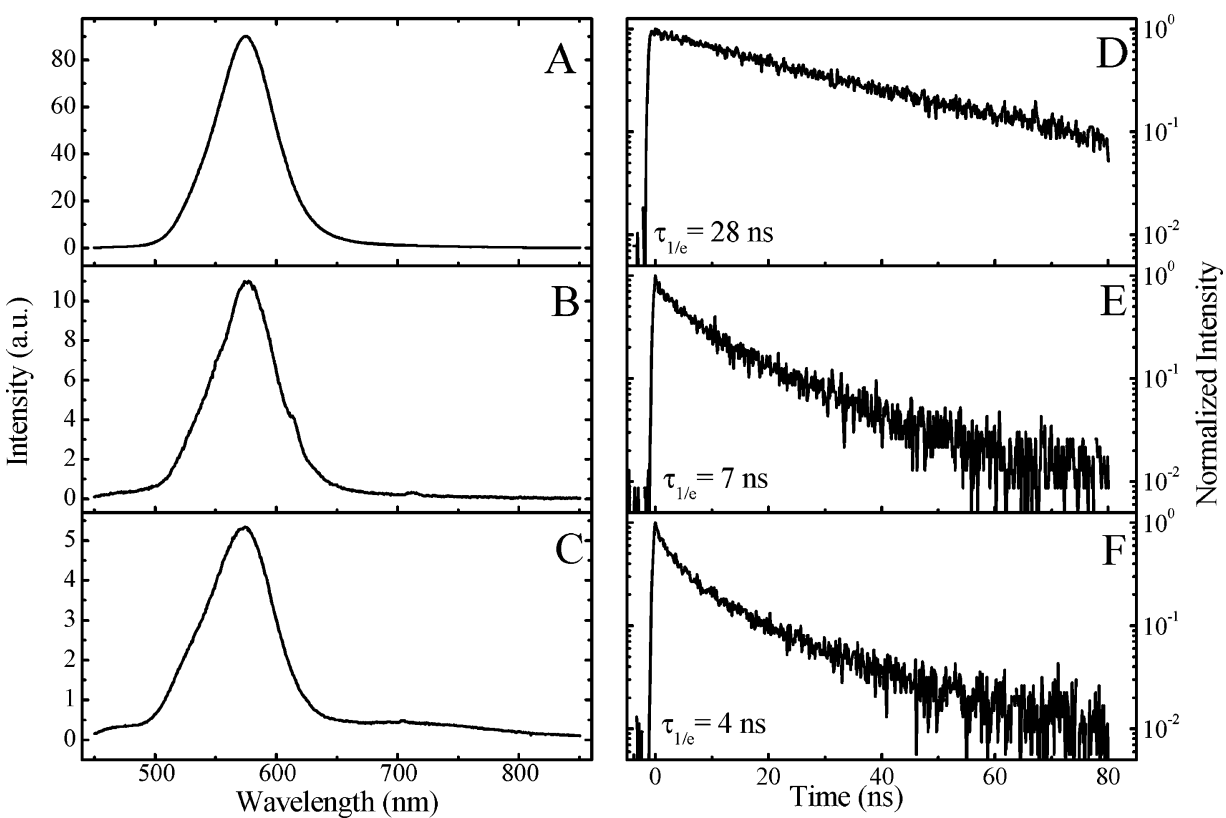

Figure 3. Emission spectra $\left(\lambda_{\mathrm{ex}}=400 \mathrm{~nm}\right)$ and normalized luminescence decay curves $\left(\lambda_{\mathrm{ex}}=400 \mathrm{~nm}\right)$ of the exciton emission of CdSe QDs capped with TOP/TOPO/HDA in chloroform (A, D), AM in chloroform (B, E), and AET in water (C, F).

The introduction of thiols to the CdSe surface has a strong effect on the luminescence QE, which drops from $55 \%$ to a few percent. Still, a weak excitonic emission is observed for the thiol-capped samples and in the case of AET-capped CdSe QDs in water also a defect-related emission is observed around 700 $\mathrm{nm}$. The luminescence decay curves (measured at the maximum of the exciton emission) of the two thiol-capped samples show a fast and strongly nonexponential decay. The decay time $\left(\tau_{1 / \mathrm{e}}\right.$ value) of the AM-capped CdSe QDs in chloroform is $7 \mathrm{~ns}$, and that of AET-capped CdSe QDs in water is $4 \mathrm{~ns}$. The fast initial decrease in the luminescence intensity also shows that fast nonradiative decay processes are induced by the thiol capping molecules.

3.3. Hole Trapping on Thiols in CdSe and CdTe Quantum Dots. Capping exchange with thiols affects the luminescence properties of CdSe QDs in a way remarkably different from that observed for CdTe QDs. Thiols that yield a high QE and a monoexponential decay for CdTe QDs are observed to almost completely quench the luminescence of CdSe (see above and also refs 12-16). This difference is particularly remarkable considering that the two II-VI semiconductors are chemically similar and have comparable band gap energies. An explanation for the strong difference between thiol-capped CdSe and CdTe QDs can be found in the energetic position of the top of the valence band. Figure 4 depicts the absolute band edge positions of bulk CdSe and CdTe in aqueous solution at $\mathrm{pH} 1 .^{23}$

Hole trapping from the semiconductor nanocrystal on a thiol is energetically favorable only if the thiol redox energy level is situated at higher energies than the valence band top. CdSe (and also $\mathrm{CdS}$ ) can very efficiently convert thiols into disulfides via a photocatalytic reaction. ${ }^{24}$ The first step in this process involves trapping of a photogenerated hole on a thiol molecule that is strongly adsorbed at the CdSe surface. Hole trapping on a thiol molecule results in the creation of a thyil radical, and two thyil radicals can be converted into a disulfide. This process is very efficient and observed not only for CdS and CdSe electrodes ${ }^{24}$ but also for CdS and CdSe QDs. ${ }^{25,26}$ Hole trapping is inhibited by an energy barrier when the top of the semiconductor valence band is above the thiol redox energy level.

The energy difference between the tops of the valence bands of bulk CdSe and CdTe is more than $0.5 \mathrm{eV}$. If the redox energy

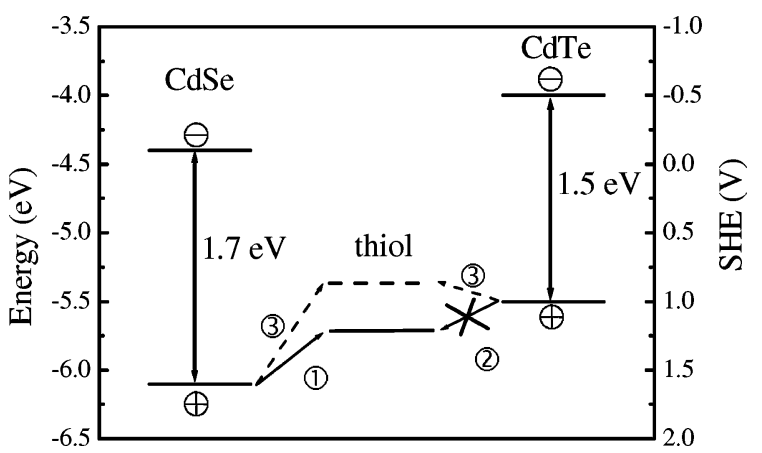

Figure 4. Positions of bulk CdSe and bulk CdTe band edges shown both on a vacuum scale and with respect to a standard hydrogen electrode reference. The standard potential expected for a thiol that does not quench the CdTe exciton luminescence is given by a solid line between $\mathrm{CdSe}$ and CdTe. Hole trapping can occur from CdSe (process (1) but not from CdTe (process (2)). The dashed line indicates the assumed position for the standard potential of a thiol that quenches the luminescence of both $\mathrm{CdSe}$ and CdTe (process (3)).

level of a thiol is situated above the top of the valence band of $\mathrm{CdSe}$ but below the top of the valence band of CdTe, an interesting situation arises (Figure 4). Hole trapping is energetically favorable for thiols attached to CdSe (process (1)) but not for CdTe (process (2)). For radiative recombination both the hole and the electron are needed. If the hole is trapped on a thiol molecule, radiative recombination of the exciton is not possible, resulting in a strongly reduced QE. If the thiol redox energy level is situated between the valence band top of bulk CdSe and CdTe, its different influence on CdSe and CdTe QDs can be explained, because this situation would induce quenching of the luminescence for CdSe QDs while increasing the QE for CdTe QDs through a better surface passivation. The observation of a weak excitonic emission for CdSe QDs capped with AET and $\mathrm{AM}$ is explained by a competition between hole trapping and radiative recombination. The rate of radiative exciton recombination is constant, whereas the hole trapping rate depends on the redox energy level of the thiol and therefore on the nature of the thiol. If the redox energy level of the thiol is higher in energy, the trapping will be energetically more favorable. This will increase the trapping rate and therefore 


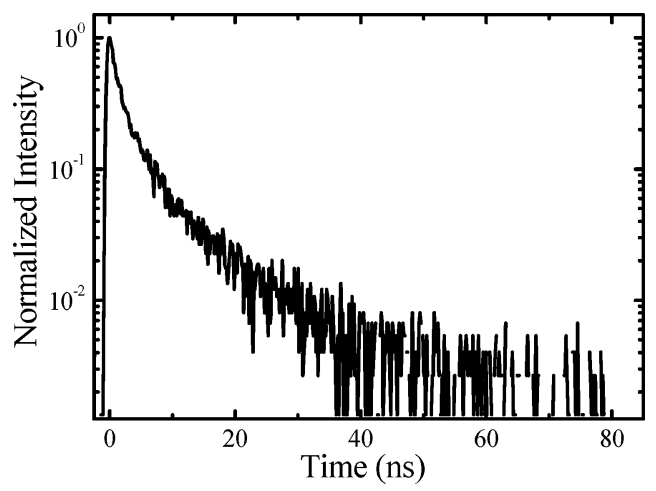

Figure 5. Luminescence decay curves $\left(\lambda_{\mathrm{ex}}=406 \mathrm{~nm}\right)$ of the exciton emission of 4-mercaptophenol-capped CdTe QDs.

decrease the quantum efficiency of the CdSe QDs. The strong initial decrease in intensity that is observed in the luminescence decay curves of the AET- and AM-capped CdSe QDs also illustrates that hole trapping is a fast process compared to radiative recombination.

For the CdTe QDs capped with, e.g., AET or AM, the exciton emission is dominated by the radiative process and is characterized by a monoexponential luminescence decay curve with a relatively long decay time $\left(\tau_{1 / \mathrm{e}} \approx 19 \mathrm{~ns}\right)$. This indicates that the hole trapping rate is low. Hole trapping is negligible if the redox energy level of the thiol is situated below the top of the valence band of CdTe (process (2) in Figure 4). The difference in energy between the top of the valence band of the two semiconductors can thus explain the large difference between the luminescence behavior of thiol-capped CdSe and CdTe QDs.

An interesting question arises if it is generally true that thiolcapped CdTe QDs show a high QE and a monoexponential luminescence decay, i.e., if hole trapping from the CdTe valence band to a thiol is always inhibited. The thiol 4-mercaptophenol is known to very efficiently quench the luminescence of CdS. ${ }^{27}$ Applying this thiol to CdSe QDs resulted in complete quenching of the luminescence (not shown). This indicates that the trapping rate of the photogenerated hole is high when 4-mercaptophenol is attached to the surface of a CdSe QD. Contrary to what would be expected on the basis of the discussion above, capping of CdTe QDs with 4-mercaptophenol decreases the luminescence intensity and leads to a strongly nonexponential luminescence decay (Figure 5). In contrast to the behavior observed for other thiols, the luminescence decay curve of 4-mercaptophenolcapped CdTe QDs is dominated by fast nonradiative processes reflected in the fast initial intensity decrease and the low QE. This observation shows that hole trapping on the 4-mercaptophenol molecules can be efficient for both CdSe and CdTe QDs (Figure 4, process (3) implying that the redox energy level of this thiol lies at higher energies than the top of the valence band for both CdSe and CdTe QDs. A reason for the higher redox energy level of 4-mercaptophenol compared to the other thiols investigated here may be the stabilization of the hole by the phenyl group of the 4-mercaptophenol molecule. This example clearly shows that the redox energy level of the type of thiol used determines the exciton decay kinetics in $\mathrm{CdSe}$ and CdTe QDs.

\section{Conclusions}

The capping of highly efficient TOP/DDA-capped CdTe QDs and TOP/TOPO/HDA-capped CdSe QDs has been exchanged with thiols containing different functional groups. For CdTe QDs capping exchange with amino ethanethiol (AET), (3-mercap- topropyl)trimethoxysilane (MPS), hexanethiol (HT), and allylmercaptan (AM) yields QDs with higher QEs and a monoexponential decay for the exciton emission. For CdSe QDs capping exchange with AET and AM results in a strong decrease in $\mathrm{QE}$ and a faster and nonexponential luminescence decay. The quenching of the exciton emission of CdSe QDs is explained by trapping of the photogenerated hole on the thiol capping molecules. The remarkable difference between thiol-capped CdTe and CdSe QDs is explained by the difference in position of the top of the valence band with respect to the redox energy level of the thiol molecule. Capping exchange with 4-mercaptophenol results in quenching of the exciton emission for both CdSe and CdTe QDs and is explained by the high redox energy level of this thiol with respect to other thiols investigated in this work.

Acknowledgment. This work was financially supported by Utrecht University within the "Breedte-strategie" program "Physics of Colloidal Matter". Dr. Peter Liljeroth is acknowledged for valuable discussions.

\section{References and Notes}

(1) Alivisatos, A. P. J. Chem. Phys. 1996, 100, 13226-13239.

(2) Brus, L. E. J. Chem. Phys. 1986, 90, 2555-2560.

(3) Peng, Z. A.; Peng, X. J. Am. Chem. Soc. 2002, 124, 3343-3353

(4) Talapin, D. V.; Rogach, A. L.; Shevchenko, E. V.; Kornowski, A.; Haase, M.; Weller, H. J. Am. Chem. Soc. 2002, 124, 5782.

(5) de Mello Donegá, C.; Hickey, S. G.; Wuister, S. F.; Vanmaekelbergh, D.; Meijerink, A. J. Phys. Chem. B 2003, 107, 489-496

(6) Bruchez, M., Jr.; Moronne, M.; Gin, P.; Weiss, S.; Alivisatos, A. P. Science 1998, 281, 2013-2016.

(7) Dubertret, B.; Skourides, P.; Norris, D. J.; Noireaux, V.; Brivanlou, A. H.; Libchaber, A. Science 2002, 298, 1759-1762.

(8) Klimov, V. I.; Mikhailovsky, A. A.; Xu, S.; Malko, A.; Hollingsworth, J. A.; Leatherdale, C. A. Eisler, H. Bawendi, M. G. Science 2000, 290, 314-317.

(9) Coe, S. Woo, W.-K. Bawendi, M. G. Bulovic, V. Nature 2002 420, 800-803.

(10) Mattoussi, H.; Mauro, J. M.; Goldman, E. R.; Anderson, G. P. Sundar, V. C.; Mikulec, F. V.; Bawendi, M. G. J. Am. Chem. Soc. 2000 122, 12142-12150.

(11) Parak, W. J.; Boudreau, R.; Le Gros, M.; Gerion, D.; Zanchet, D.; Micheel, C. M.; Williams, S. C.; Alivisatos, A. P.; Larabell, C. Adv. Mater. 2002, 14, 882-885.

(12) Talapin, D. V.; Rogach, A. L.; Mekis, I.; Haubold, S.; Kornowski, A.; Haase, M.; Weller, A. Colloids Surf., A 2002, 202, 145-154.

(13) Wuister, S. F.; Swart, I.; van Driel, F.; Hickey, S. G.; de Mello Donegá, C. Nano Lett. 2003, 3, 503-507.

(14) Rogach, A. L.; Kornowski, A.; Gao, M.; Eychmüller, A.; Weller, H. J. Phys. Chem. B 1999, 103, 3065-3069.

(15) Gaponik, N.; Talapin, D. V.; Rogach, A. L.; Hoppe K.; Shevchenko, E. V.; Kornowski, A.; Eychmüller, A.; Weller, H. J. Phys. Chem. B 2002 $106,7177-7185$.

(16) Gao, M.; Kirstein, S.; Möhwald, H.; Rogach, A. L.; Kornowski, A.; Eychmüller, A.; Weller, H. J. Phys. Chem. B 1998, 102, 8360-8363.

(17) Talapin, D. V.; Haubold, S.; Rogach, A. L.; Kornowski, A.; Haase, M.; Weller, A. J. Phys. Chem. B 2001, 105, 2260-2263.

(18) Wuister, S. F.; Van Driel, F. Meijerink, A. Phys. Chem. Chem. Phys. 2003, 5, 1253-1258.

(19) Ebenstein, Y.; Mokari, T.; Banin, U. Appl. Phys. Lett. 2002, 80, 4033-4035.

(20) Fisher, B. R. Eisler, H. J. Stott, N. E.; Bawendi, M. G. J. Phys. Chem. B 2004, 108, 143-148.

(21) Wuister, S. F.; de Mello Donegá, C.; Meijerink, A. J. Chem. Phys. 2004, 121, 4310-4315.

(22) Kuno, M.; Lee, J. K.; Dabbousi, B. O.; Mikulec, F. V.; Bawendi, M. G. J. Chem. Phys. 1997, 106, 9869-9882.

(23) Hagfeldt, A.; Graetzel, M. Chem. Rev. 1995, 95, 49-68.

(24) Natan, M. J.; Thackeray, J. W.; Wrighton, M. S. J. Phys. Chem. 1986, 90, 4089-4098.

(25) Fischer, C.-H.; Henglein, A. J. Phys. Chem. 1989, 93, 5578-5581.

(26) Aldanan, J. Wang, Y. A. Peng, X. J. Am. Chem. Soc. 2001, 123, $8844-8850$

(27) Tata, M.; Banerjee, S.; John, V. T.; Waguespack, Y.; McPherson, G. L. Colloids Surf. A 1997, 127, 39-46. 